\title{
Improved Aerodynamic Prediction Through Coupled System and CFD Models
}

\author{
Ed Tate $^{1}$ Joaquin Gargoloff ${ }^{1}$ Brad Duncan ${ }^{1}$ \\ Hubertus Tummescheit ${ }^{2}$ John Griffin ${ }^{2}$ John Batteh ${ }^{2}$ \\ ${ }^{1}$ Exa, USA, \{edtate, joaquin, brad\} @exa.com \\ ${ }^{2}$ Modelon, USA, \{hubertus.tummescheit, john.griffin, john.batteh\}@modelon.com
}

\begin{abstract}
Accurate predictions of aerodynamic forces using computational fluid dynamics require accurate geometry. The aerodynamic forces on the vehicle body affect the vehicle posture or the vehicle position with respect to the ground. When a vehicle is cruising on the road, the change in vehicle posture is usually relatively small with respect to the size of a vehicle. However, these small changes in geometry can lead to significant differences in aerodynamic drag and airflow structures. To address this issue, a coupled simulation approach was developed to predict vehicle posture in typical cruise and wind tunnel test conditions. This coupled approach was tested using Exa's PowerFLOW and Modelon's Vehicle Dynamics Library (VDL). In this approach, the aerodynamic forces on the body are used to calculate the movement of the body and the suspension geometry. This modified geometry is then used to recalculate the operating aerodynamic forces. The modified geometry shows changes in total force, the distribution of forces, and the structure of the airflow over the vehicle. The results provided by correct geometry under loaded conditions offer better correlation to test and provide car makers with the increased accuracy to confidently improve real world fuel economy.
\end{abstract}

Keywords: aerodynamics, suspension, co-simulation

\section{Introduction}

One of the most important aspects of a vehicle for fuel economy is the aerodynamic drag. Reducing drag improves fuel economy in conventional vehicles and range in electric vehicles. When a new vehicle is designed, a car maker must decide where to invest resources in meeting mandated and customer expected efficiency requirements. Meeting efficiency targets usually involves improving drag, reducing powertrain losses, and reducing vehicle mass. Improvements in each of these areas represent significant investments on any new program. Accurately predicting the drag is critical to predicting the performance that a production vehicle will achieve. If this value is accurately predicted, an OEM can confidently direct the large investments associated with improving fuel economy and range. If this value is incorrectly predicted, then late design changes that carry a large risk and expense are needed to meet the original vehicle targets. Predicting vehicle efficiency involves many tools that are used for simulating the different aspects of a vehicle. The vehicle drag prediction requires $3 \mathrm{D}$ CFD simulation. The efficiency is usually predicted in system simulations that consider drag, body, and powertrain behavior.

Two common assumptions are used when determining drag for fuel economy, range, and vehicle dynamics simulations. The first is that vehicle aerodynamic forces are accurately represented by a load curve that is a function of vehicle speed. The second is that vehicle geometry is fixed for characterizing aerodynamic forces. Both assumptions are valid, but only for limited conditions. In both the wind tunnel and the real world, these assumptions reduce the accuracy of the resulting predictions.

In a system simulation, the effect of aerodynamic forces on a vehicle is usually calculated using the coefficient of drag. This coefficient is determined from a CFD simulation, measured in a wind tunnel, or derived from a coast down test. For fixed geometry in still air, the drag force, $F_{D}$, is a function of the square of the vehicle speed, $v$, the air density, $\rho$, the coefficient of drag, $C_{D}$, and the frontal area of the vehicle.

$$
F_{D}=\frac{1}{2} \cdot \rho \cdot v^{2} \cdot C_{D} \cdot A
$$

The drag force works against the direction of travel of a vehicle. However, in addition to the drag force, the airflow over the vehicle also generates lift forces. These lift forces cause the posture of the vehicle to change, with a 2 millimeter front ride height increase and a 3 millimeter rear ride height for our example, as illustrated in Figure 1. The lift force is calculated similar to the drag force using a lift coefficient. To calculate the vehicle posture, lift forces are calculated over the front and rear axles using an equation similar to the drag equation. 


$$
\begin{aligned}
F_{L, \text { Front }} & =\frac{1}{2} \cdot \rho \cdot v^{2} \cdot C_{L, \text { Front }} \cdot A \\
F_{L, \text { Rear }} & =\frac{1}{2} \cdot \rho \cdot v^{2} \cdot C_{L, \text { Rear }} \cdot A
\end{aligned}
$$

The lift is determined in this way so that the effect of different lift forces on the front and rear of the vehicle are considered. The lift driven changes in posture mean that the assumption of fixed geometry doesn't hold. Therefore, for accurate prediction of forces on a vehicle, this interaction between vehicle posture and aerodynamic forces should be considered. CFD accuracy is improved by considering the impact of aerodynamic forces on vehicle posture.

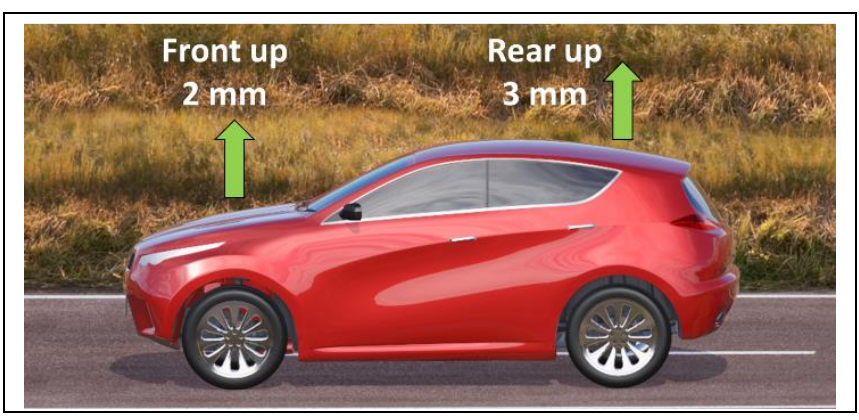

Figure 1. Lift forces and displacement.

\section{Determining Posture Change}

Changes in vehicle posture affect the position and orientation of suspension parts and wheels. These small changes in geometry affect the airflow over the entire vehicle. The changes in airflow change the pressure on the vehicle surfaces. This change in the pressure distribution and magnitude cause a change in the lift and drag forces. In some cases, this change in posture has an easily observable effect on the airflow. For example, a part of the underbody which was shielded from high speed airflow might be exposed and act like an aeronautic air brake. In other cases, the effect may be subtle, causing changes in the distribution of the flow over the vehicle body and relative change in the flow under and over the vehicle. This effect is similar to how changing an airfoil's angle of attack changes its lift and drag. A key difference is that a vehicle's geometry is much more complex than an airfoil. It has complex surfaces, heat exchangers, fans, airflow through the engine bay, rotating tires, and airflow around the vehicle body.

To accurately determine the effect of these geometry changes a full 3D flow simulation is required. This simulation is done using the Lattice-Boltzmann (LB) solver in PowerFLOW (Exa Corporation, 2017). This solver offers several advantages over traditional Navier-Stokes (NS) based solvers. The LB solver is inherently a transient solver, and the PowerFLOW implementation is able to handle fully detailed automotive geometry without simplification. This ability to handle geometry changes without special consideration simplifies implementation of geometry movement. This solver is used by OEM's globally for aerodynamic, thermal, and acoustic simulation. Its accuracy and robustness are well documented (Kotopati, 2009; Duncan, 2010; Duncan, 2012).

Modelica was used in this application because it is capable of describing problems in many engineering domains. Most importantly, it can elegantly describe multi-body problems such as suspension simulations. The features inherent in the language make it easy to present the model in a form that can be used by someone who is not an expert in a particular engineering domain, such as suspension simulation.

Furthermore, since Modelica is able to address multiple engineering domains, it provides a solution to describing different functional behavior in the vehicle using a single language. Vehicle Dynamics Library (VDL) (Modelon AB, 2016) has been used extensively in the automotive domain and proven for simulation of complex vehicle behavior (Andreasson, 2011; Andreasson, 2016; Griffin, 2012; Klomp, 2016) in Dymola (Dassault Systemes, 2017). VDL is a commercial Modelica library with a wide range of full fidelity, multibody suspension configurations. VDL can solve for the effect of aerodynamic load, like in a wind tunnel or the open road, and inertial loads, like on the track. In conjunction with OPTIMICA Compiler Toolkit (OCT) (Modelon AB, 2016), Functional Mockup Units (FMUs) (MODELISAR, 2010) from VDL can be created to simply the task of interfacing between multiple solvers.

A model of a proprietary vehicle from Exa known as the EV12 was implemented using the Vehicle Dynamics Library (VDL) from Modelon. The EV12 vehicle has a McPherson strut front suspension and a twist beam rear suspension. As suspension topologies are available in VDL, modelling the EV12 was simply a matter of modifying the suspension geometry parameters to match those of the EV12. The resulting vehicle model in Dymola is shown in Figure 2. 


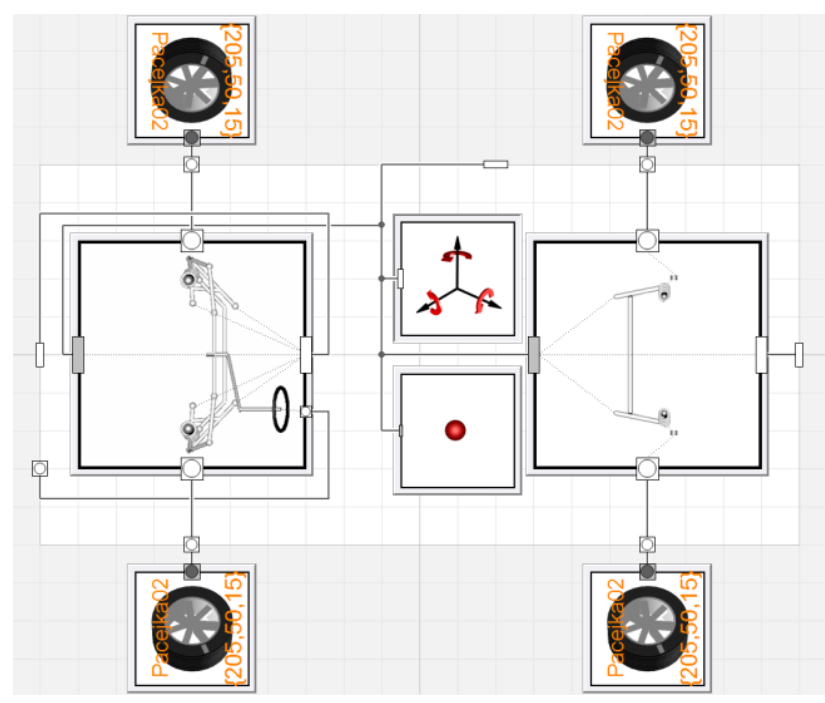

Figure 2. Diagram view of EV12 chassis model

Two different approaches were used during to the investigation to quantify the difference in drag forces and vehicle pressure distribution.

\subsection{Change in vehicle posture based on downforce}

In the first approach, the goal was to determine the effect changes in aerodynamic forces had on the static vehicle posture.

To quantify this effect, the vehicle posture was controlled by aerodynamic downforce. As changes in downforce directly relate to changes in tire vertical forces, tire vertical forces were used to resolve the ride height. This change was implemented as a controller in the system model.

The ride height controller, shown in Figure 3, was implemented by defining the fender height, or vertical height of the chassis at each vehicle corner, versus tire vertical force as tabular data and adjusting the force in the actuator until the desired fender height was achieved. The tire vertical force is a standard output in VDL for vehicle simulations. Therefore, accessing the tire vertical force to use it in the actuator was simply a matter of pulling this signal off the signal Bus. A PIDcontroller from the Modelica Standard Library was used to control the force.

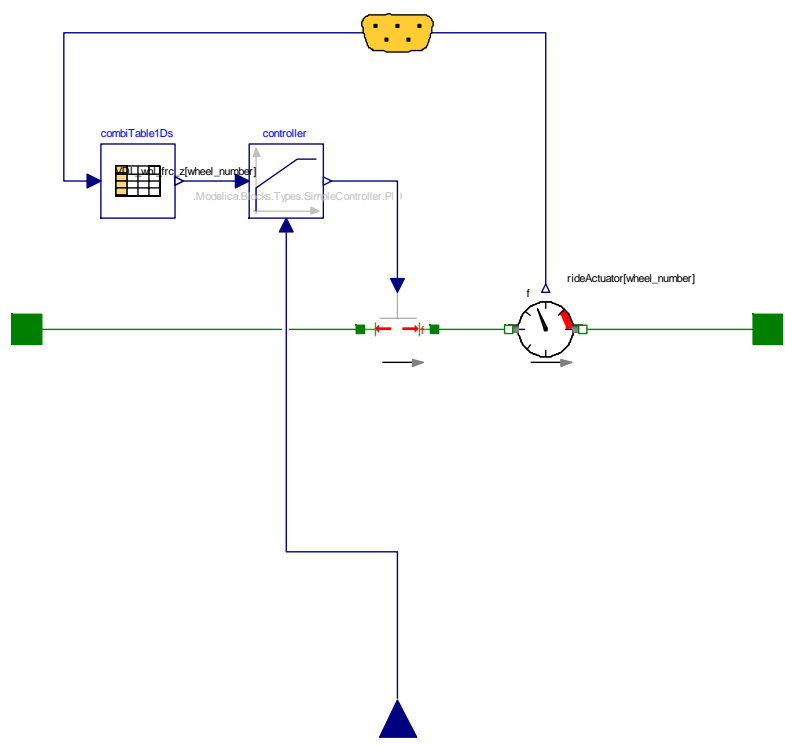

Figure 3. Ride height controller

Control of the vehicle posture was achieved by using ride height actuators as shown in Figure 4. VDL uses both standardized templates and interfaces to describe vehicle components and sub-components. The ride height controller described above used a consistent interface as the standard ride springs. As such changing from the standard ride spring model to ride height actuator was simply a matter of changing classes.

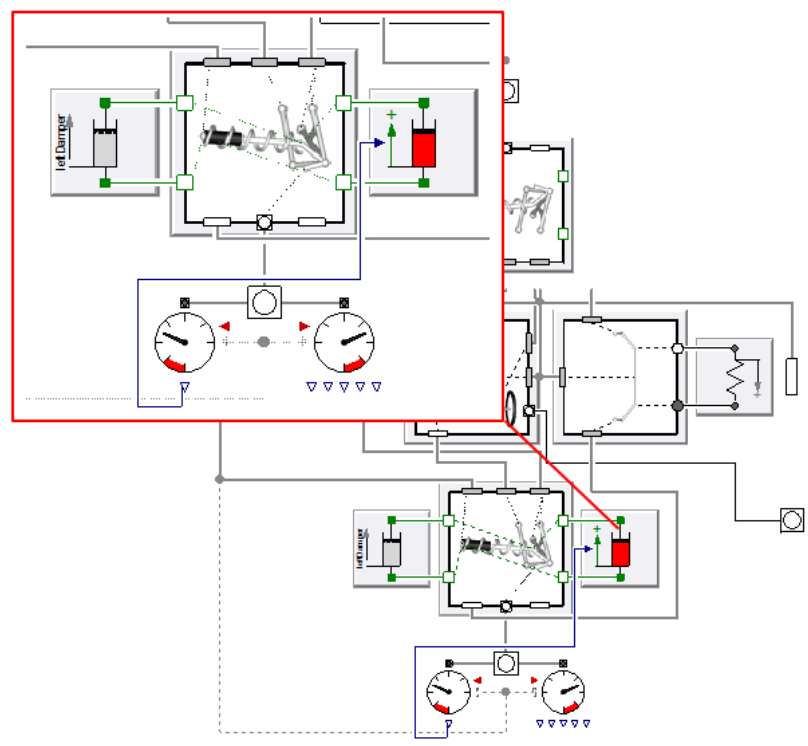

Figure 4. Standard ride springs replaced with actuators

Based on this approach, we concluded that even small changes in aerodynamic downforce affected both vehicle posture and the position and orientation of the suspension components. 


\subsection{Controlled vehicle posture}

In the second approach, the vehicle posture was explicitly controlled.

The vehicle posture was changed using a standard experiment in VDL in which the wheel hubs are held at a fixed vertical position and the chassis is pulled down by two actuators. The attachment points of the actuators on the vehicle were located at positions consistent with the sensors that measure the front and rear ride height in the CFD simulation. The diagram layer of the heave rig experiment is shown in Figure 5.

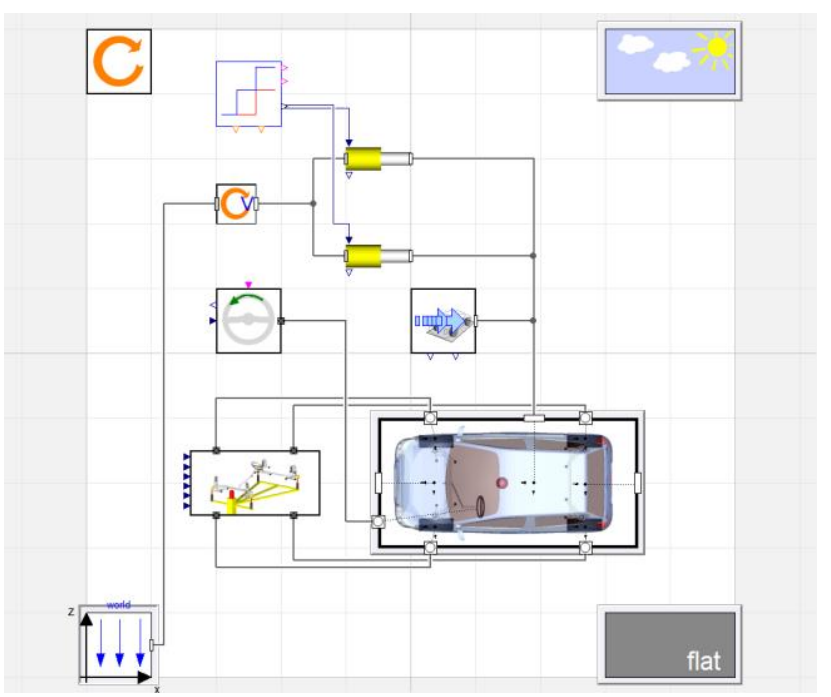

Figure 5. Diagram layer of HeaveRig experiment

The resulting animation of the heave rig experiment is shown in Figure 6.

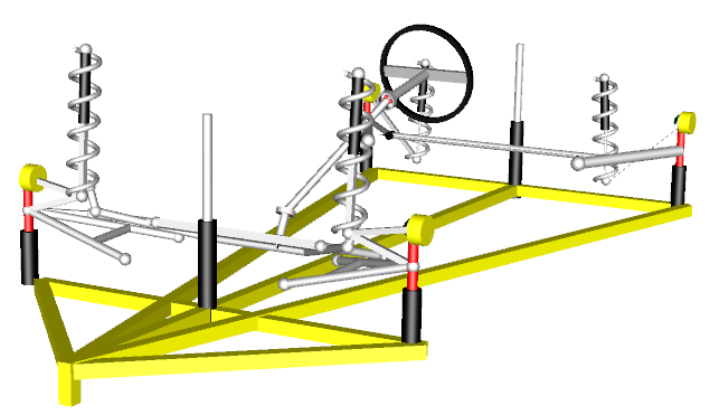

Figure 6. Animation of HeaveRig experiment

The desired results of the heave rig were the time history of all suspension part positions and orientations at all front and rear right heights. To generate this data, a full variable sweep was used in which the front and rear ride heights were varied from -15 to $15 \mathrm{~mm}$ of travel at $1 \mathrm{~mm}$ intervals. This full sweep resulted in 961 different vehicle postures.

As is evidenced in Figure 7, the suspension components of the vehicle move significantly across the various vehicle postures. The image below was generated by superimposing all the animation frames.

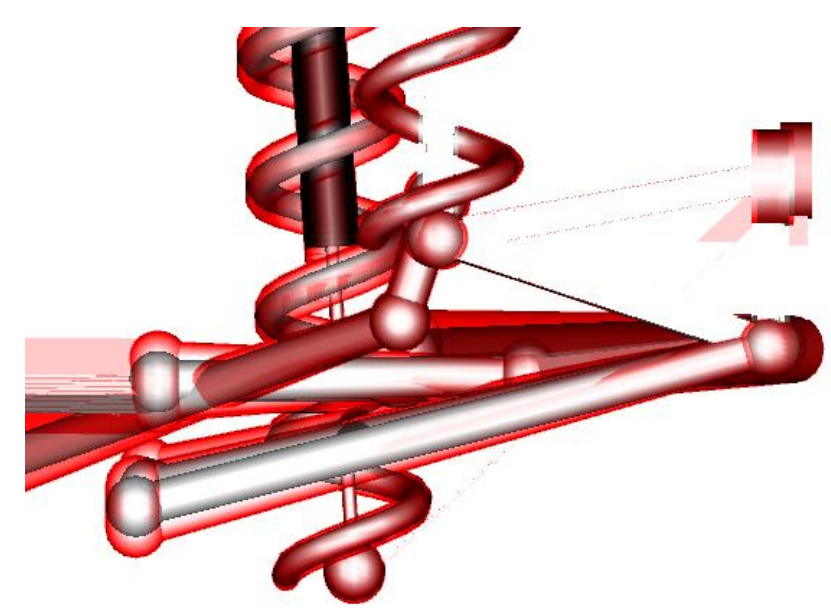

Figure 7. Superimposed frames of HeaveRig animation

The overall magnitude of the change in suspension component position and orientation is shown in Figure 8 . This plot shows the change in the height of the outer tierod point vs. front and rear heave changes.

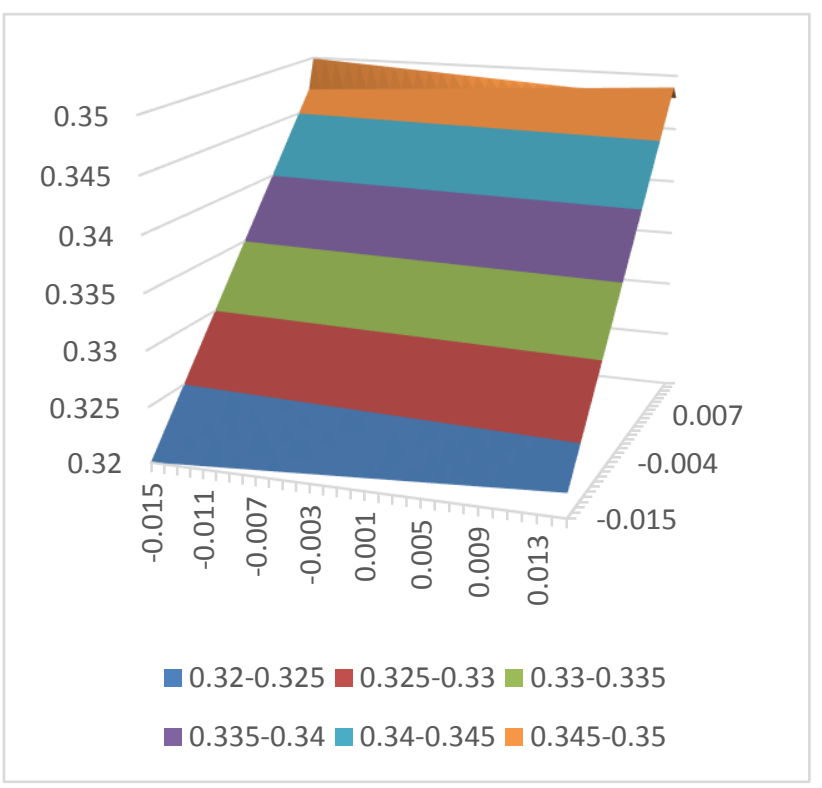

Figure 8. Variation of outer tierod height with changes in vehicle posture

The HeaveRig simulation provided a complete time history of suspension parts position and orientation during the vehicle posture changes. These results were exported and reformatted for use in PowerFLOW.

\section{Improved Drag Prediction}

The most important design point for a vehicle's aerodynamics is the performance under steady speed conditions on a flat road. This condition is the one 
replicated in most wind tunnels. When operating in this manner, the vehicle's change in posture is caused by the drag force and the lift forces. When the vehicle posture changes, many components in the suspension move. This movement is illustrated in Figure 9. Most importantly, the vehicle body position changes.

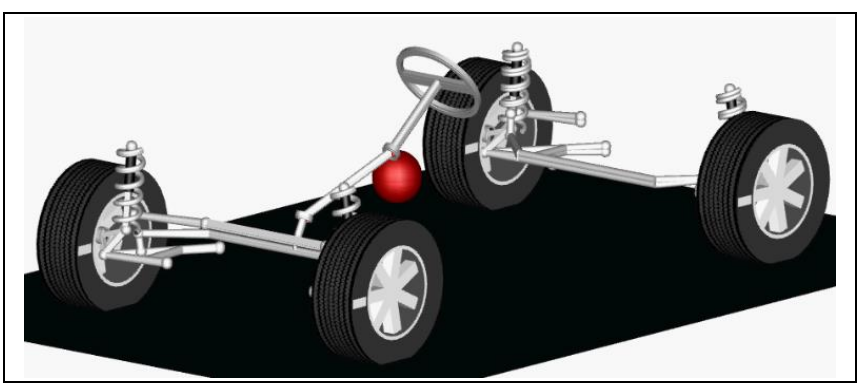

Figure 9. Suspension displacement under aerodynamic load.

While small changes in posture of a few millimeters may appear to be inconsequential, these effects are often a source of error for accurately predicting the drag of a vehicle. Furthermore, these small changes in posture lead to appreciable changes the flow structures on the vehicle. Such an effect is illustrated in Figure 10.

The change in the vehicle posture exposes the front suspension to more incoming flow, which increases the static pressure on the surface of the vehicle, increasing drag. This effect (higher static pressure) is visible in both the lower A-arm attachment to the body as well as the front wheel arch pressure, behind the front suspension. These two areas are marked with white arrows on each image of Figure 10. Both areas show a redder shade of static pressure, contributing to about 1 count of aerodynamic drag (a count is 0.001 or a tenth of a percent).

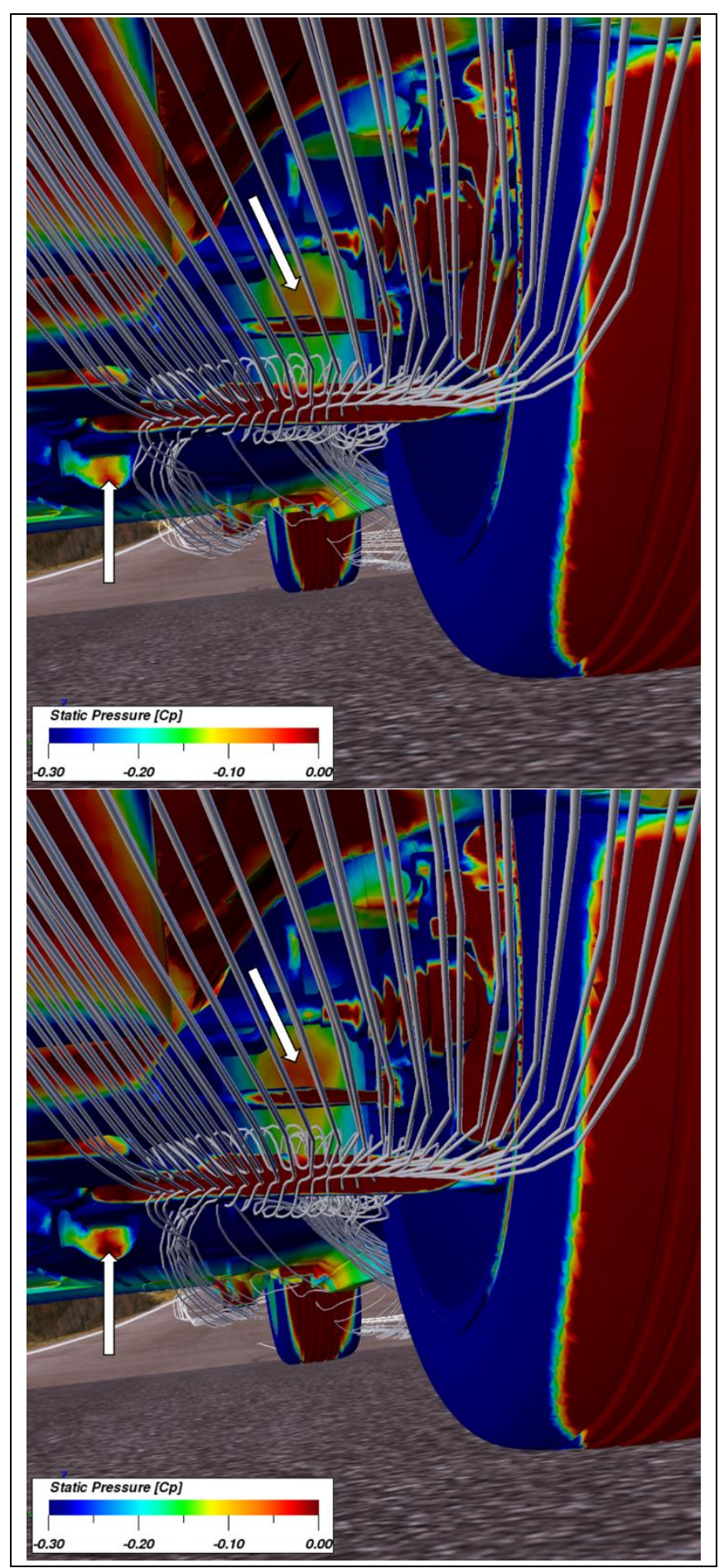

Figure 10. Differences in surface pressure and underbody airflow. Original posture [top] vs. realistic posture [bottom]

Focusing on the rear of the vehicle, Figure 11 shows the surface pressure for both the baseline vehicle (top) as well as the realistic posture (bottom). It can be seen that updating the posture and the suspension yield a lower surface pressure on the back of the vehicle, which contributes to 3 count of drag increase. 

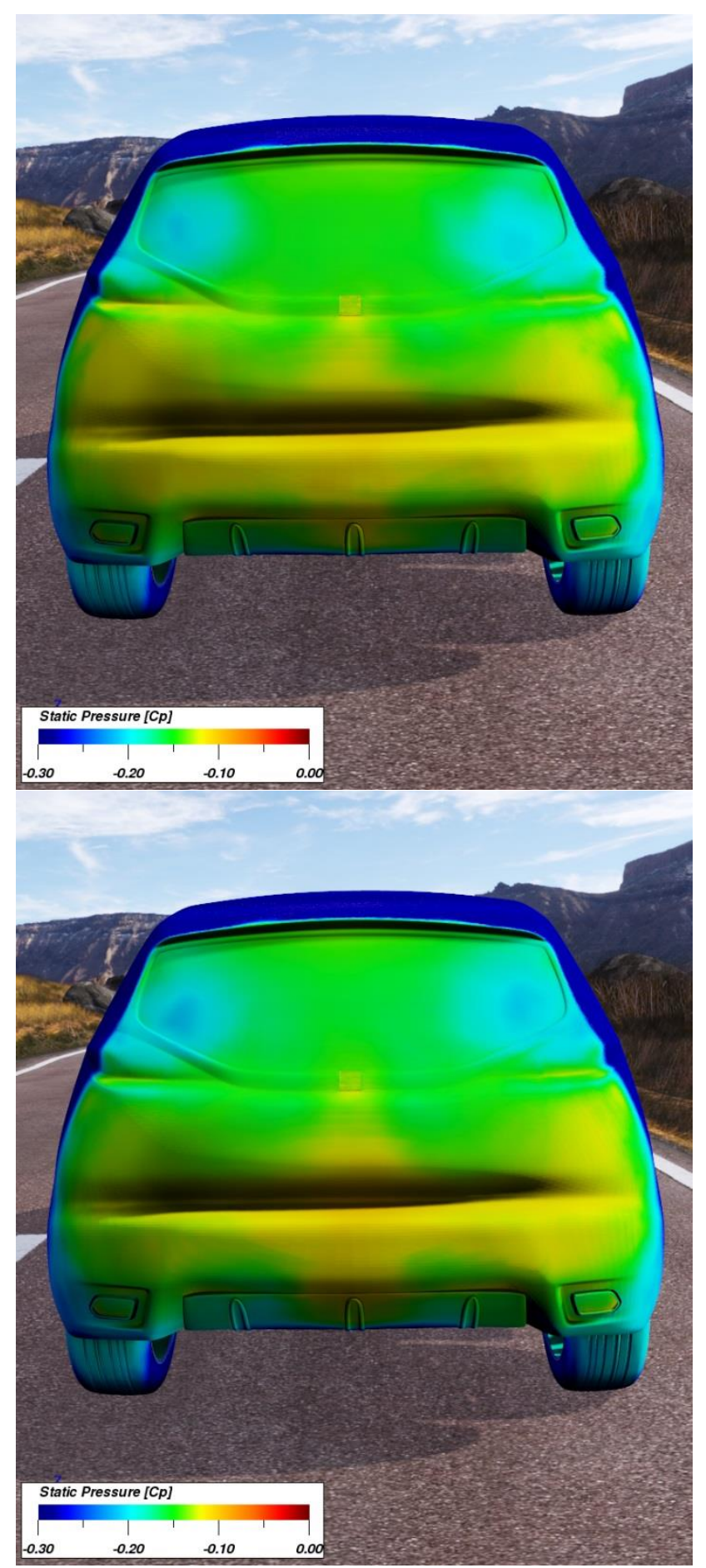

Figure 11. Differences in surface pressure on the back of the vehicle. Original posture [top] vs. realistic posture [bottom]

In this case study, this coupling improved the accuracy of the drag predictions by 4 counts, about a $1 \%$ improvement. Simulating a step in between (updating the body posture alone without updating the suspension) enabled us to find that of the 4 total counts of drag increase, 3 were due to the body posture update and 1 count was due to updating the suspension geometry. These effects can be seen in Table 1 .

\begin{tabular}{|l|c|c|}
\hline Case: & Cd [counts] & $\Delta$ Cd [counts] \\
\hline Baseline & 387 & \\
\hline Posture alone & 390 & +3 \\
\hline Posture+Suspension & 391 & +4 \\
\hline
\end{tabular}

Table 1. Difference in vehicle drag due to posture and suspension change.

This improved accuracy was achieved by first finding the drag and lift forces on the vehicle using the at-rest posture. This model included all the vehicle geometry details such as underhood and suspension components. After finding the lift forces, they were applied to the suspension model described in Section 2.1 .

The change in posture resulted in changes in the airflow pressure on the vehicle body. Using the corrected geometry, these refined forces are a more accurate representation of the vehicle forces and flows. The local impact of the changes can be seen in the drag development show in Figure 12. This graph shows that the body posture effect of 3 counts is mainly felt at the back of the vehicle, manifesting in a reduction in the base pressure. The suspension effects, on the other hand, have a 1 count of drag impact that is felt mainly on the front axle suspension and the front wheel arches.

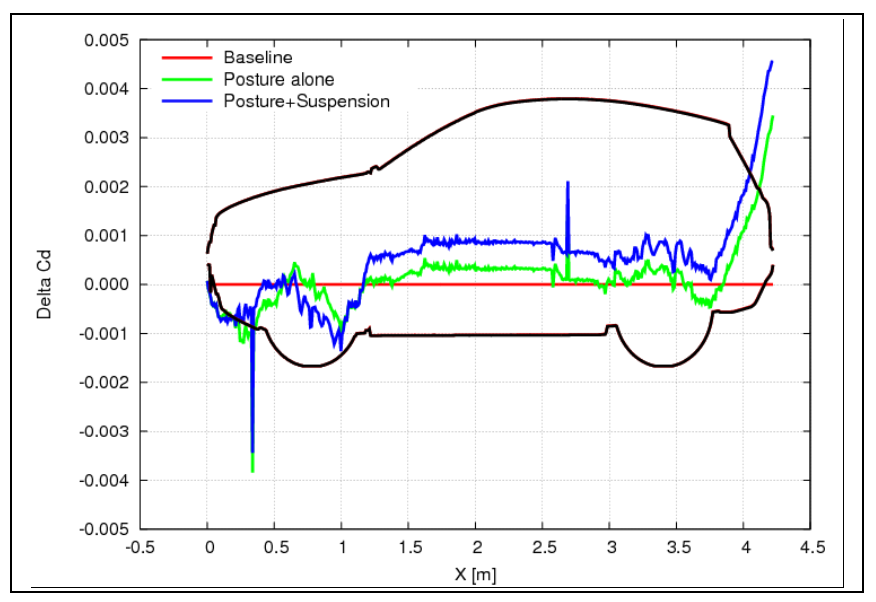

Figure 12. Difference in drag due to posture change.

This iterative coupling solved for the vehicle lift, posture change, and then for the improved drag value. The inputs to the process are the vehicle geometry and the suspension characteristics. The vehicle geometry provides the surface data for CFD simulation and the location of the hard points in the suspension which are used to setup the system simulation. The suspension characteristics allow the system model to properly calculate the changes in geometry due to the lift forces. 


\section{A Case Study}

This process was applied to a proprietary vehicle from Exa known as the EV12. This vehicle has dimensions and details similar to a small SUV. To calculate the posture change, Vehicle Dynamics Library was used to determine the vehicle geometry changes in response to these forces. These changes in geometry were used to modify the vehicle geometry. This modified vehicle geometry was used to find an updated drag value. For this case study, the need to iterate on this process was investigated. It was found that a single iteration was sufficient to account for posture driven changes in drag. This quick convergence allows improved drag prediction at an affordable computational and walltime cost.

The system model was integrated in a rig which simulates the body motion in response to the drag and lift forces. This rig is shown in Figure 4. The remaining part of this solution is the translation of the suspension movement back to changes in geometry. This translation is accomplished by using consistent frames of reference between the system model and the CFD model. The changes of the frames of reference are determined in response the aerodynamic forces.

\section{Conclusions}

A methodology was developed that improves the correlation of vehicle geometry to real world loading conditions and thus improves accuracy in replicating test conditions in CFD. This improvement was accomplished by coupling a Vehicle Dynamics Library model of the vehicle with Exa's PowerFLOW for 3D CFD simulation. The vehicle model was coupled with the CFD simulation via an FMU generated using OPTIMICA Compiler Toolkit.

In the case study considered, changes in drag of about $1 \%$ were seen due to the changes in vehicle posture and consequently changes to the suspension geometry. Improving aerodynamic prediction accuracy is critical because of the large impact on certification and real world fuel economy. This case study examined a single aspect of coupling aerodynamic and suspension simulations. This coupling is important enough that it is expected to be part of every vehicle aerodynamic simulation. Future applications will consider the impact of real world conditions, tire tread, and driving cycles to improve designs for efficiency and comfort.

\section{References}

Andreasson, J., “The Vehicle Dynamics Library: New Concepts and New Fields of Application", Proceedings of 8th International Modelica Conference, 2011.

Andreasson, J., Machida, N., Tsushima, M., Griffin, J., Sundström, P.: Deployment of high-fidelity vehicle models for accurate real-time simulation. Japanese Modelica Conference 2016, Tokyo, Japan, May 23-24, 2016.

Dassault Systemes, Velizy, France (2017) Dymola 2017 FD01. https://www.3ds.com/productsservices/catia/products/dymola/

Duncan BD, Fischer A, and Kandasamy S.: Validation of lattice-Boltzmann aerodynamics simulation for vehicle lift prediction. In: ASME 2010 3rd joint US-European fluids engineering summer meeting, 8th international conference on nanochannels, microchannels, and minichannels, Montreal, Quebec, Canada, 1-5 August 2010, ASME paper FEDSM-ICNMM2010-30891, pp. 2705-2716. New York: ASME.

Duncan B, Kandasamy S, Gau H, et al.: Aerodynamic performance assessment of BMW validation models using computational fluid dynamics. SAE paper 2012-01-0297, 2012.

Exa Corporation, Burlington, Mass, USA (2017) PowerFLOW. http://exa.com/en/product/simulationtools/powerflow-cfd-simulation/

Griffin, J., Batteh, J., and Andreasson, J., "Modeling Vehicle Drivability with Modelica and the Vehicle Dynamics Library", Proceedings of 9th International Modelica Conference, pp. 599-608, 2012.

Klomp, M., Sundström, P., Johnsson, A.: Real-Time Simulation of Elasto-kinematic Multi-body Vehicle Models. 13th International Symposium on Advanced Vehicle Control, Munich, Germany, pp. 255-260, Sep. 1316, 2016.

Kotopati R, Keating A, Kandasamy S, et al.: The latticeBoltzmann-VLES Method for automotive fluid dynamics simulation, a review. SAE paper 2009-26-057, 2009.

MODELISAR, Functional Mock-up Interface for Model Exchange, Version 1.0, 2010.

Modelon AB, Lund, Sweden. (2017). OPTIMICA Compiler Toolkit. http://www.modelon.com/products/optimicacompiler-toolkit/

Modelon AB, Lund, Sweden. (2017). Vehicle Dynamics Library. $\quad$ http://www.modelon.com/products/modelicalibraries/vehicle-dynamics-library/ 